Original Article

\title{
Thermal Effects on Fluid Mixing in the Eye
}

\author{
Jinglin Huang (i) and Morteza Gharib \\ Department of Medical Engineering, California Institute of Technology, 1200 E California Blvd, MC 105-50, Pasadena,
} CA 91125, USA

(Received 27 December 2019; accepted 12 May 2020; published online 26 May 2020)

Associate Editor Arash Kheradvar oversaw the review of this article.

\begin{abstract}
Age-related macular degeneration (AMD) is the leading cause of central vision loss in the developed world. Wet AMD can be managed through serial intravitreal injections of anti-vascular endothelial growth factor (antiVEGF) agents. However, sometimes the treatment is ineffective. Given that the half-life of the drug is limited, inefficient mixing of the injected drug in the vitreous chamber of the eye may contribute to the ineffectiveness. Here, we introduce thermal heating as a means of enhancing the mixing-process in the vitreous chamber and investigate parameters that potentially influence its effectiveness. Our in vitro studies reveal the importance of the heating location on the eye. A significant increase in the mixing and delivery of drugs to the targeted area (the macula) could be achieved by placing heating pads to induce a current, against gravity, in the vitreous. The presented results can potentially help in the development of a better strategy for intravitreal injection, subsequently improving the quality of patient care.
\end{abstract}

Keywords-Vitreous, Age-related macular degeneration (AMD), Particle imaging velocimetry (PIV), Drug delivery, Intravitreal injections, Thermally-induced convection, Flow in the human eye.

\section{INTRODUCTION}

The human eye is a complex physiological machine, with delicate flow dynamics that maintain its health and intended optical function. ${ }^{5,7}$ About $80 \%$ of its space is the vitreous chamber, which sits between the lens and the retina. The vitreous chamber is filled with a fluid-like gel, vitreous humor, that is important for performing various treatments in the eye (Fig. 1a). Vitreous humor contains approximately $98-99 \%$ water

Address correspondence to Jinglin Huang, Department of Medical Engineering, California Institute of Technology, $1200 \mathrm{E}$ California Blvd, MC 105-50, Pasadena, CA 91125, USA. Electronic mail: alicehuang0904@gmail.com with trace amounts of hyaluronic acid, glucose, ions, and collagen. ${ }^{15}$ The eye comprises three layers: the outer layer, which includes the sclera and the cornea; the middle layer, which includes the iris, the choroid, and the ciliary body; and the inner layer, which includes the retina. ${ }^{13}$ The retina is the light-sensitive nerve layer that senses light and sends impulses through the optic nerve to the brain to enable vision. The macula is a small but important area in the center of the retina. It has a high concentration of optical nerves that facilitates fine, detailed vision. ${ }^{14}$ During the aging process, the macula encounters diverse issues that can lead to severe vision loss if proper treatment is not received. Macula-related diseases include branch retinal vein occlusion, macular degeneration (dry or wet), retinal detachment, and retinitis pigmentosa.

Among these diseases, age-related macular degeneration (AMD) is the leading cause of central vision loss in the developed world. ${ }^{6,21}$ Patients with AMD see a dark spot in the center of their vision. There are two forms of the disease. The dry form is characterized by yellow deposits or choroidal neovascularization that develops underneath the retinal pigment epithelium. Fluid and blood leak through these abnormal vessels, creating scar tissue. In the wet form, normal macular tissues are lost, causing severe vision loss. ${ }^{6,19}$ Wet AMD can be managed through serial intravitreal injections of anti-vascular endothelial growth factor (anti-VEGF) agents, where an ophthalmologist injects $50 \mu \mathrm{L}$ of the drug into the vitreous chamber of a patient every few weeks. ${ }^{20}$ This treatment method can also be used to treat diseases such as diabetic macular edema, retinal vein occlusion, and other vascular disorders.

Because the drug delivery mechanism in the eye of each individual is not well understood, ophthalmolo- 
(a)

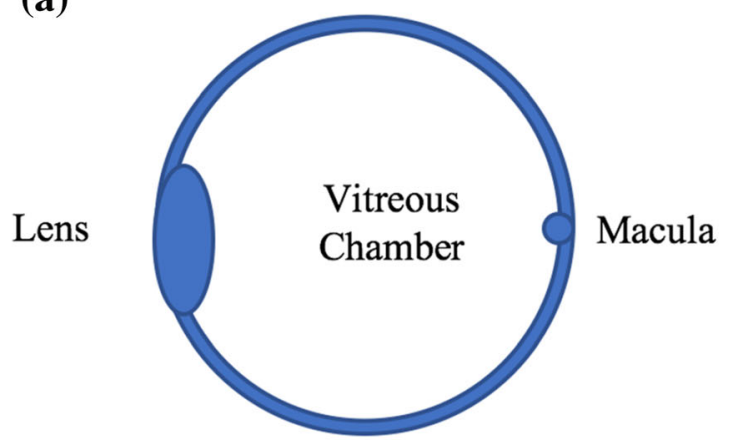

(b)

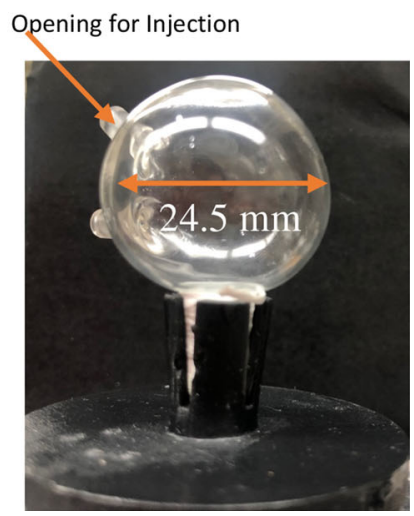

FIGURE 1. Eye model: a $24.5 \mathrm{~mm}$ diameter quartz globe. (a) Basic schematic of the eye. (b) Eye model with two vertically aligned openings for injection. One opening intersects the globe equator, and the other has a $45^{\circ}$ angle to the equator. Both openings are about $0.5 \mathrm{~mm}$ in diameter, and the average globe thickness is about $1 \mathrm{~mm}$.

gists can only determine whether another injection is needed based on an empirical method, the observation of improvements in the patient's visual acuity. ${ }^{22}$ For patients whose visual acuity does not significantly improve, another intravitreal injection is administered. The variation in treatment effectiveness among patients remains unclear. Furthermore, these injections may cause complications such as intraocular inflammation, retinal detachment, traumatic lens damage, and sustained ocular hypertension. ${ }^{8,16}$

Inefficient drug delivery to the macula could be one cause of the ineffectiveness of the treatment. The drug can be transported in the eye either by pure diffusion or by convection current. ${ }^{17}$ The timescale for pure diffusion is typically larger than that of convection current. For anti-VEGF agents, the typical diffusion coefficients (dc) values ${ }^{3,11}$ range from 20 to $1200 \mu \mathrm{m}^{2}$ $\mathrm{s}^{-1}$. For a typical human eyeball of $25.4 \mathrm{~mm}$ in diameter $(D=25.4 \mathrm{~mm})$, the timescale for the drug to cross the vitreous chamber by pure diffusion, estimated by $\tau \sim D^{2} / \mathrm{dc}$, ranges from 6 to 120 days. Because the half-life of anti-VEGF agents is quite limited, ${ }^{3,10}$ innovative methods are needed to expedite the drug transport process to ensure that more of the injected high-potency dose reaches the target tissue. Increasing drug delivery efficiency during each injection can potentially reduce both the frequency of injections and the required dose, thereby alleviating possible complications.

Components of human vitreous change consistently as people age. At birth, the vitreous is a $100 \%$ gel solution, which liquefies gradually as people age. ${ }^{1}$ AMD affects the population of age 50 and older the most. Eye morphology also varies among different individuals, which affects the density/viscosity of vitreous. This could be another reason for the discrepancy in treatment efficacy among various individuals.
In this work, we investigate the thermally induced convection approach for enhancing drug mixing and transport in the vitreous chamber. The study objectives are to (1) effectively induce convection flow in the eye to accelerate drug transport efficiency, and (2) study the efficacy of the induced circulation in facilitation of the drug reaching the targeted area. It is worth noting that there has not been much study of the role of thermally induced convection in promoting drug mixing in the eye previously. The results of the current study can not only provide a simple approach to be incorporated into clinical procedures for improved patient care but also offer insight into flow dynamics in the vitreous chamber of the human eye. We investigate the effects of the following three parameters: (1) temperature difference between the eye and the heat source, (2) heating position on the eye, and (3) density/ viscosity difference between the drug and vitreous humor.

\section{MATERIALS AND METHODS}

\section{Eye Model Fabrication and Vitreous Humor Preparation}

We used an eye model in the form of a 1 inch $(24.5 \mathrm{~mm})$ diameter glass globe (Fig. 1b). We considered the fact that components of human vitreous change with age. To determine the range of physical values of human vitreous properties, we refer to the work of Murthy, ${ }^{12}$ which states that the viscosity of human vitreous is two to four times greater than water. This conclusion is derived based on a study conducted by John Locke, M.D., and Ross Morton, M.D. ${ }^{9}$ Based on these facts, we chose glycerol/water mixtures at various ratios for our vitreous humor model in the experiments.

\section{BMES


Glycerol and water at various weight ratios (glycerol in 20.2, 33.6, and $11.2 \mathrm{wt} \%$ ) were made in bulk. Each set of mixtures was placed at room temperature for at least $48 \mathrm{~h}$ to ensure a consistent initial temperature. A summary of the properties of the materials used and a comparison with the properties of human vitreous can be found in Table 1. $20.2 \mathrm{wt} \%$ glycerol mixture was determined to be the primary working fluid to serve as the vitreous humor model in our study.

\section{Heat Source and Heating Positions}

The heat source for inducing a temperature gradient was composed of a steel rod, a polyimide flexible heater (OMEGA Engineering, KHLVA-0502), an aluminum piece, and a power supply. The aluminum piece was customized to have a curved surface to fit the surface of the eye model, making sure that the effective contact area of the heat source was approximately $5 \mathrm{~mm}$ by $5 \mathrm{~mm}$. For the upright head position, three locations were selected for the heating elements to be applied, as shown in Fig. 2a. We also studied the effect of heating for face-down and face-up positions, as shown in Figs. $2 \mathrm{~b}$ and $2 \mathrm{c}$, respectively. Depending on the heating element locations, two temperature differences, measured as the initial temperature difference between the heating element and the eye model, $\triangle T=10{ }^{\circ} \mathrm{C}$ and $\triangle T=5{ }^{\circ} \mathrm{C}$, were applied. The heating element temperature was kept constant throughout the experiment by applying a constant voltage from the power supply. The selected temperature range is safe for applications on human eyes, ${ }^{18}$ as some commercial eye heating masks already have temperature settings that exceed this range [such as Aroma Season moist heated eye mask for stye blepharitis treatment with flaxseed, which has a heating range from $40\left(104^{\circ} \mathrm{F}\right)$ to $\left.60{ }^{\circ} \mathrm{C}\left(140^{\circ} \mathrm{F}\right)\right]$. Before running the actual experiments, we used the same setup to monitor temperature variation in $1 \mathrm{~h}$ with the aid of an FLIR SC6700 infrared camera (equipped with FLIR Exam IR software) and an Omega Engineering HH806AU thermometer (equipped with surface thermocouple with self-adhesive backing, Type k). The overall eye model temperature increase was around $2{ }^{\circ} \mathrm{C}$.

\section{Visualization Techniques}

Particle imaging velocimetry (PIV) visualization and laser-induced fluorescence (LIF) visualization were used in this study. For both visualization techniques, a thin laser sheet was generated and applied to visualize the center plane of the eye model. A convex lens was used to spread the laser beam vertically so that the camera visualized only a thin sheet of the entire eye model, as shown in Fig. 3a. The laser beam was carefully calibrated to pass through the center of the eye model. Essentially, flow profiles on a 2D plane at the center of a 3D object were observed. In our study, a 445-nm blue laser was used to illuminate the particleladen flow field (laser model: Skye 100-mW handheld 445-nm focusable blue laser pointer with dual lock). The flow images were captured by a camera with a resolution of 640 by 480 pixels (IPX-VGA210-L; $90 \mathrm{~mm}$ Tamron Lens, Saitama, Japan) at 10 frames per second (fps). The camera setting was adjusted using the free software LYNX GigE Application.

For PIV visualization, 80- $\mu \mathrm{m}$ fluorescence particles were mixed with $20.2 \mathrm{wt} \%$ glycerol mixture for qualitative and quantitative velocity and vorticity field visualization in the eye model (Fig. 3b). The PIV analysis was performed using commercial software, PIV3Cview (PIVTEC), to obtain velocity profiles. During each PIV visualization session, a total of 6000 images were taken $(10 \mathrm{~min}$ at $10 \mathrm{fps})$, which were segregated into 60 groups, with 100 images in each group. The 100 particle images in each group were overlaid in sequence onto a single image, which resulted in one slice of stacked images for each group. A total of seven groups of particle pathline visualizations were summarized for each analysis. A sample velocity field is shown in Fig. 3b, from which we calculate the circulation of the flow. Circulation is calculated by taking the integral of vorticity across the entire plane of observation. The average circulation intensities in the plane of observation at different time

TABLE 1. Properties of the vitreous and model materials used in this study.

\begin{tabular}{lccccc}
\hline Material & $\begin{array}{c}\text { Viscosity } \\
(\mathrm{mPa} \mathrm{s})\end{array}$ & $\begin{array}{c}\text { Density } \\
\left(\mathrm{kg} \mathrm{m}^{-3}\right)\end{array}$ & $\begin{array}{c}\text { Thermal expansion } \\
\text { coefficient }\left(20^{\circ} \mathrm{C}\right)\end{array}$ & $\begin{array}{c}\text { Coefficient of } \\
\text { Refractive } \\
\text { index }\end{array}$ & $\begin{array}{c}\text { thermal conductivity } \\
\left(20^{\circ} \mathrm{C}\right)\left(\mathrm{W} \mathrm{m}^{-1} \mathrm{~K}^{-1}\right)\end{array}$ \\
\hline Human vitreous & $1-3.6$ & $1005.3-1008.9$ & - & 1.337 & 0.594 \\
$20.2 \mathrm{wt} \%$ glycerol & 1.7 & 1046.8 & $3.2 \times 10^{-4}$ & 1.357 & 0.556 \\
$33.6 \mathrm{wt} \%$ glycerol & 2.7 & 1081.5 & $3.9 \times 10^{-4}$ & 1.375 & 0.515 \\
$11.2 \mathrm{wt} \%$ glycerol & 1.3 & 1024.5 & $2.6 \times 10^{-4}$ & 1.346 & 0.591 \\
\hline
\end{tabular}


Gravity

(a)

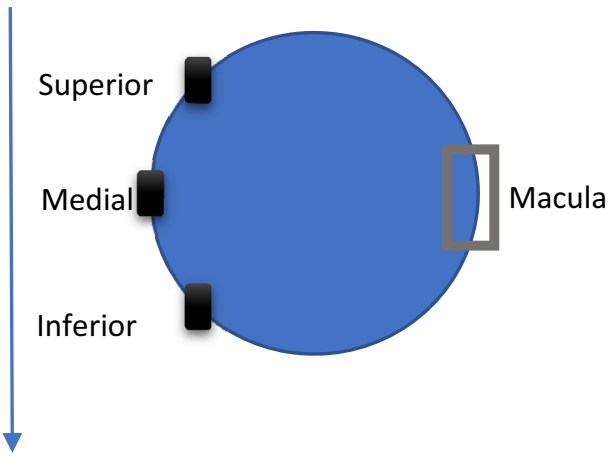

(b)

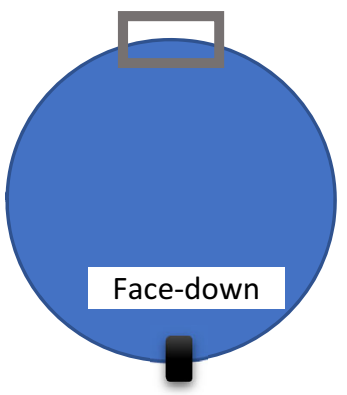

(c)

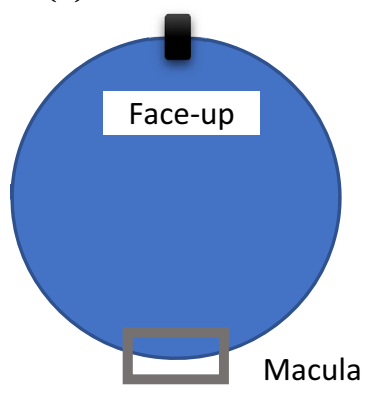

FIGURE 2. The five heating positions used in this study: (a) sitting/standing in the upright position, (b) facing down, and (c) facing up. Positions of the heater are marked by the black squares and the position of the target tissue, the macula, is marked by the hollow grey square.

(a)

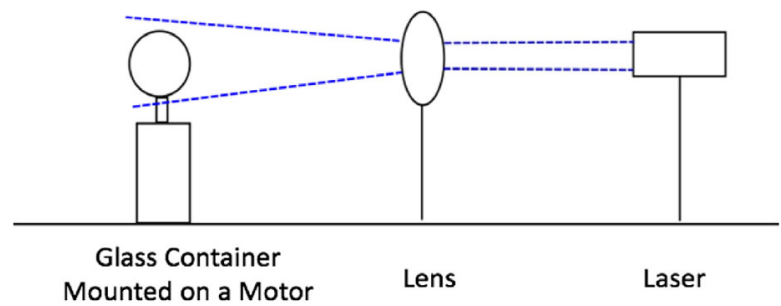

(b)

(c)
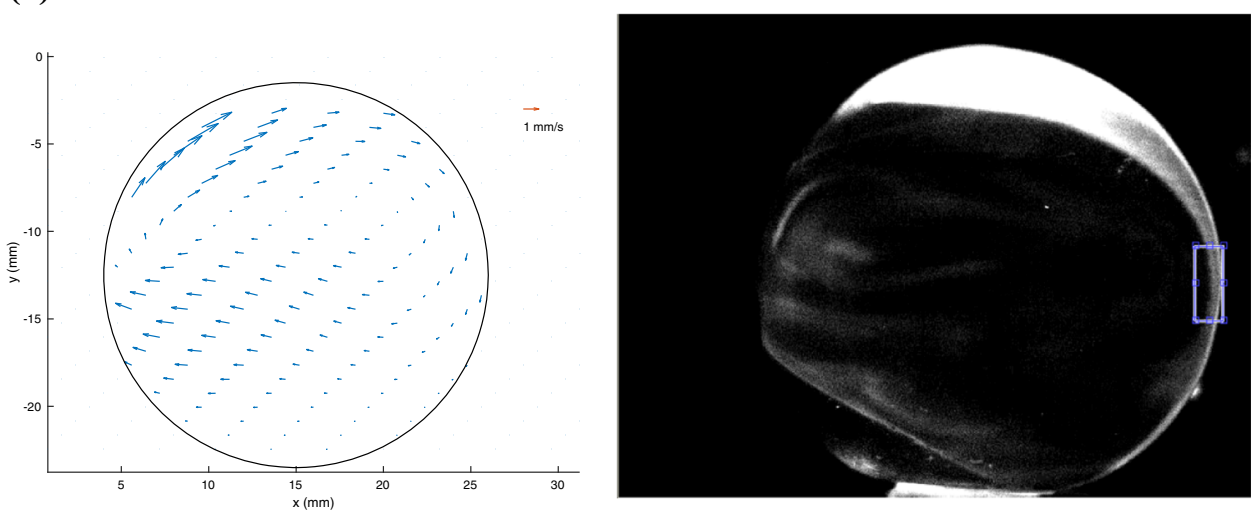

FIGURE 3. Particle imaging velocimetry (PIV) and laser-induced fluorescence (LIF) visualization setup. (a) Schematic of experimental setup. The convex lens expands the laser beam into a thin sheet positioned at the center plane of the eye model. (b) A typical velocity field representation obtained from the PIV analysis (the sample image shows the velocity field from $\triangle T=10^{\circ} \mathrm{C}$ medial heating after heater is ON for $5 \mathrm{~min}, x$ and $y$ reflect the length and height in the field of view). (c) Pixel intensity quantification by MATLAB. The purple box indicates the area of interest for pixel intensity quantification. When fluorescein is excited by the laser, it emits bright/white in the image. In this image, fluorescein is distributed in the top right region.

points throughout the heating process were calculated and summarized.

For LIF visualization, the $20.2 \mathrm{wt} \%$ glycerol mixture was the primary working solution. The 11.2 and $33.6 \mathrm{wt} \%$ glycerol mixtures were each mixed with an aqueous fluorescein solution $(0.2 \mathrm{~g} / \mathrm{mL})$ at a $1: 50$ solution-to-mixture volume ratio to produce models for a drug that is lighter and less viscous than the vitreous and a drug that is heavier and more viscous that the vitreous, respectively. The introduction of the latter mixture to the eye model using a 27-gauge needle simulated the process of adding a drug that was heavier and more viscous than the vitreous humor. Correspondingly, adding the former mixture to the eye model simulated the process of adding a lighter and less viscous drug to the eye. The LIF visualization was performed for the following four cases, all with $\Delta T=10^{\circ} \mathrm{C}$ : (a) $33.6 \mathrm{wt} \%$ glycerol for the drug sim- 
ulant and $20.2 \mathrm{wt} \%$ glycerol as the vitreous mixture medial heating, (b) $33.6 \mathrm{wt} \%$ glycerol for the drug simulant and $20.2 \mathrm{wt} \%$ glycerol as the vitreous mixture inferior heating, (c) $11.2 \mathrm{wt} \%$ glycerol for the drug simulant and $20.2 \mathrm{wt} \%$ glycerol as the vitreous mixture medial heating, and d) $11.2 \mathrm{wt} \%$ glycerol for the drug simulant and $20.2 \mathrm{wt} \%$ glycerol as the vitreous mixture inferior heating.

The total observation time was 50 min and images were sampled at 0,10 , and $50 \mathrm{~min}$. Drug simulant was injected into the eye model using a 27 -gauge syringe needle. To quantify the amount of drug (glycerol/water/fluorescein mixture) that reached the target region, MATLAB was used for pixel intensity quantification (Fig. 3c). The area of interest was defined as a 5-pixel by 20-pixel rectangle located at a typical position of the macula in the human eye. The size and location of this region were kept the same throughout all image quantifications.

\section{RESULTS}

\section{PIV Visualization and Particle Pathline (Overall Velocity Field)}

PIV visualization results are shown in Figs. 4 and 5. Experiments were conducted for five heating positions and two temperature differences and compared with a control case in which the heater was off (Fig. 4a).

PIV analysis provided information about velocity and vorticity fields at different times throughout the heating process. The absolute values of the average circulation intensities, calculated from the vorticity across the entire plane of observation, at six different time points throughout the heating process are summarized in Fig. 5. The qualitative information in Fig. 4 and the corresponding circulation values in Fig. 5 provide information about the strength of the convectively induced currents for each case.

Time-averaged images for the control case without heating in Fig. 4a depict the baseline. Figure $4 \mathrm{~b}$ shows the case for medial heating with $\triangle T=5^{\circ} \mathrm{C}$, in which the formation of a double-circulation region on the left half of the eye model produces voids in regions near the targeted macula area. Also, we note from Fig. 5 that the strength of circulation remains low but constant up to $600 \mathrm{~s}$ of observation. In the case for medial heating with $\triangle T=10^{\circ} \mathrm{C}$ shown in Fig. $4 \mathrm{c}$, the formation of similar double-circulation regions covers the entire midplane of the eye model. We also note the presence of flow activity in the vicinity of the targeted area. Figure 5 indicates that the strength of the circulation for this case also remains constant with time but with values three times higher than those in the $\triangle T=5{ }^{\circ} \mathrm{C}$ case.
The inferior heating case shows a dramatic deviation from the medial heating case. For example, for inferior heating with $\triangle T=10^{\circ} \mathrm{C}$, we note formation of a single rotational region by $20 \mathrm{~s}$ after heating starts, followed by the appearance of a weaker secondary rotational region in the subsequent $5 \mathrm{~min}$ (Fig. 4d). Accordingly, Fig. 5 depicts an increasing trend of circulation with time for inferior heating, which eventually reaches a constant level after $400 \mathrm{~s}$. For this case, one can see strong flow parallel to the walls near the targeted area for the entire observed time.

It is interesting to note that for the same $\triangle T=10$ ${ }^{\circ} \mathrm{C}$, this dramatic flow activity subsides substantially when the heater is moved to the superior position, as shown in Fig. 4e. Corresponding circulation values (Fig. 5) show a substantial reduction compared to the values in the inferior heating case. This superior heating case would be similar to the case of a person who is lying down with his/her head facing up (Fig. 4f) and placing a heat source on top of his/her eyes. Therefore, one should not expect much flow activity.

Also, corollary to the case of inferior heating with $\triangle T=10^{\circ} \mathrm{C}$, one can imagine a person in a face-down position with a heating pad in the medial position (Fig. 4g). This case shows an even higher circulating region in terms of flow and circulation strength. Circulation values reach up to seven times higher than those of the upright medial heating case (Fig. 5). It is notable that for this case, we did not reach the equilibrium value within our observation time period.

The general observation of all the cases that we have studied indicates that heating from the inferior position (against the gravity) induces the strongest circulation motion. For both inferior heating in the upright position (Fig. 4d) and face-down heating (Fig. 4g), strong flow and circulation were present over the entire eye model.

The specific nature of the circulation patterns for each case leads us to expect different outcomes for the movement of injected drug. To investigate examples of this effect, we used the velocity field obtained by PIV to track a circular 2D disc (radius $=0.4 \mathrm{~mm}$ ) scalar pattern (as representative of an injected drug) for three scenarios: medial heating with $\triangle T=5{ }^{\circ} \mathrm{C}$, medial heating with $\triangle T=10^{\circ} \mathrm{C}$, and inferior heating with $\Delta T=10^{\circ} \mathrm{C}$. The results are summarized in Fig. 6 . For a $2 \mathrm{D}$ analysis, we kept the initial position of the scalar circle in all three scenarios the same and the goal was to observe the time it took for the particle to reach the back of the eye during the 10-min heating process. The position of the macula is in the back of the eye, which sits on the right boundary of the circular domain in our model. Because particles tend to move along the curvature once they are near the boundary, we assume 
(a)



(b)

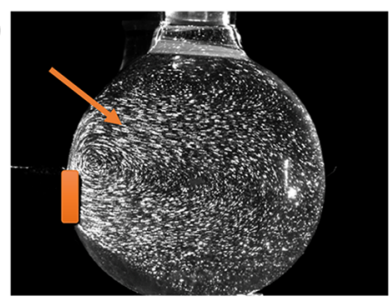

(c)



(d)



(e)

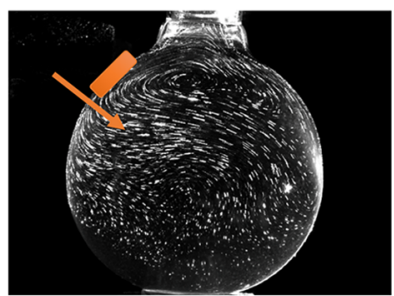

(f)

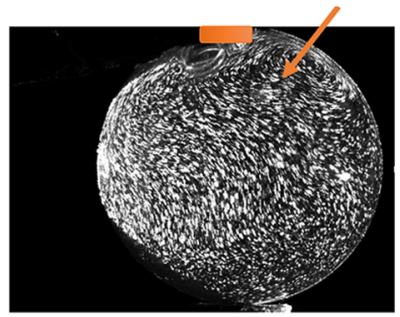

(g)

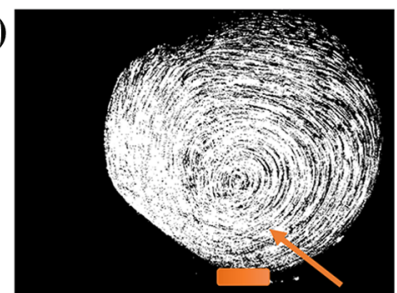

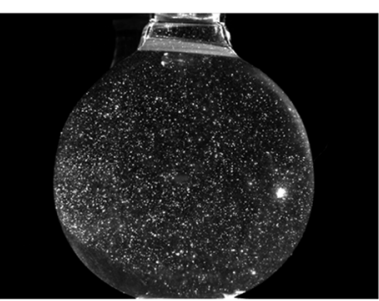
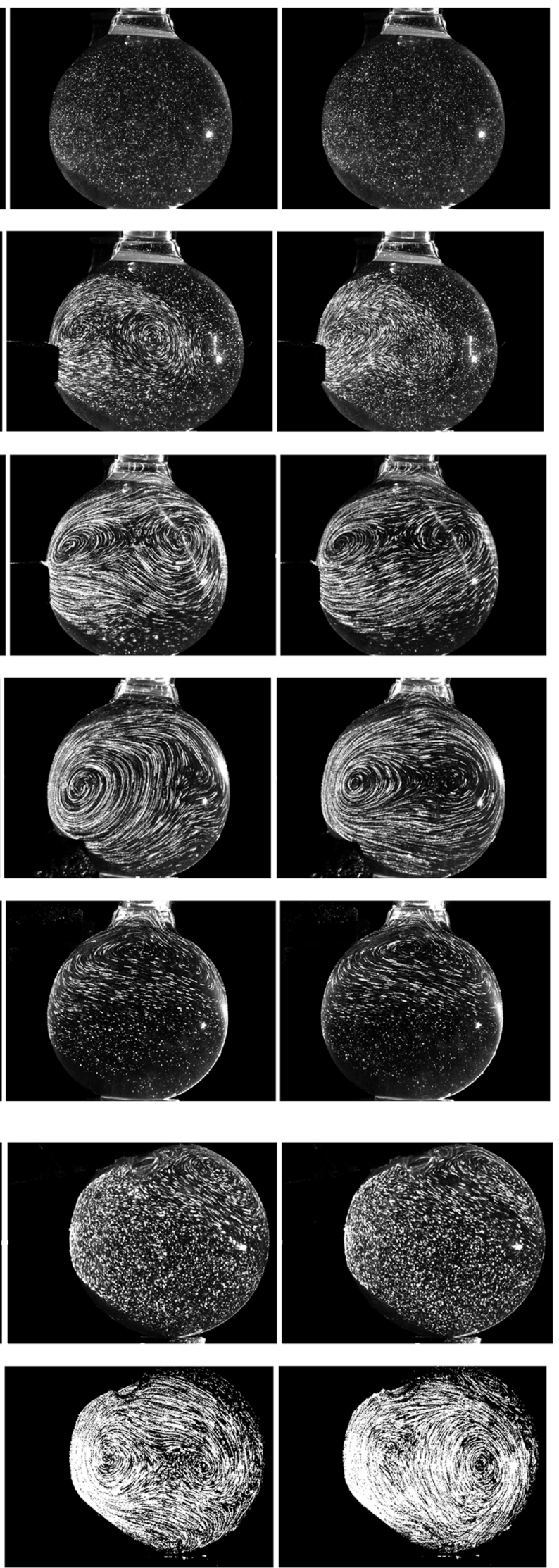

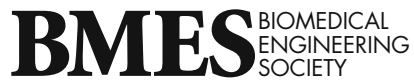


4FIGURE 4. A summary of particle pathline visualizations for seven different heating scenarios at three time points (left to right, $10-20 \mathrm{~s}, 5-5 \mathrm{~min} 10 \mathrm{~s}$, and $9 \mathrm{~min} 50 \mathrm{~s}-10 \mathrm{~min}$ after the heater is turned on): (a) No heating (heater off). (b) Medial heating with $\triangle T=5^{\circ} \mathrm{C}$. (c) Medial heating with $\triangle T=10^{\circ} \mathrm{C}$. (d) Inferior heating with $\triangle T=10^{\circ} \mathrm{C}$. (e) Superior heating with $\triangle T=10^{\circ} \mathrm{C}$. (f) Face-up heating with $\triangle T=10^{\circ} \mathrm{C}$. (g) Facedown heating with $\triangle T=10^{\circ} \mathrm{C}$. Note that locations of heating and injection are artificially marked with orange block and arrow, respectively.

that the scalar (drug) delivery is successful as soon as it reaches the boundary on the right.

As demonstrated in Fig. 6, heating from an inferior position at $\triangle T=10{ }^{\circ} \mathrm{C}$ helped the center of the scalar pattern to reach the back of the eye in less than $200 \mathrm{~s}$. The center of the scalar disc for the case of heating from the medial position at $\triangle T=10^{\circ} \mathrm{C}$ was trapped in a flow loop during the first $400 \mathrm{~s}$ and eventually started to move toward back of the eye after $600 \mathrm{~s}$ (10 min). For the case of heating from the medial position at $\triangle T=5{ }^{\circ} \mathrm{C}$, no part of the scalar disc reached the back of the eye. These observations are consistent with the observations from the previous pathline visualizations.

In summary, inferior position heating for a person in the upright position as well as heating from a facedown position can induce very strong flow circulation, which is the goal. The strong circulation induced by these heating positions can help facilitate efficient drug mixing in the eye after an intravitreal injection. To understand whether the drug was actually "delivered" (i.e., reached the macula) given a strong circulation motion, we simulated the actual drug delivery process in vitro for inferior and medial position heating with $\triangle T=10{ }^{\circ} \mathrm{C}$, and found that the results were consistent with the PIV visualization results.

\section{LIF Dye Pattern Visualization}

We used experimental qualitative LIF visualization of dye patterns to elucidate the effect of density and viscosity difference between an injected drug and the vitreous media. In Fig. 7, LIF flow images are shown for the four tested cases. The selected time history of the average pixel intensity reading of the dye arriving in the targeted area, determined by the method described in the experimental methods section, is summarized in Fig. 8.

When drug simulant (shown by fluorescent dye) was heavier and more viscous than the vitreous (Figs. 7a and $7 b$ ), it initially stayed at the bottom of the eye model. When heating was applied from the medial position (Fig. 7a), drug gradually moved upward but hardly reached the targeted region throughout the

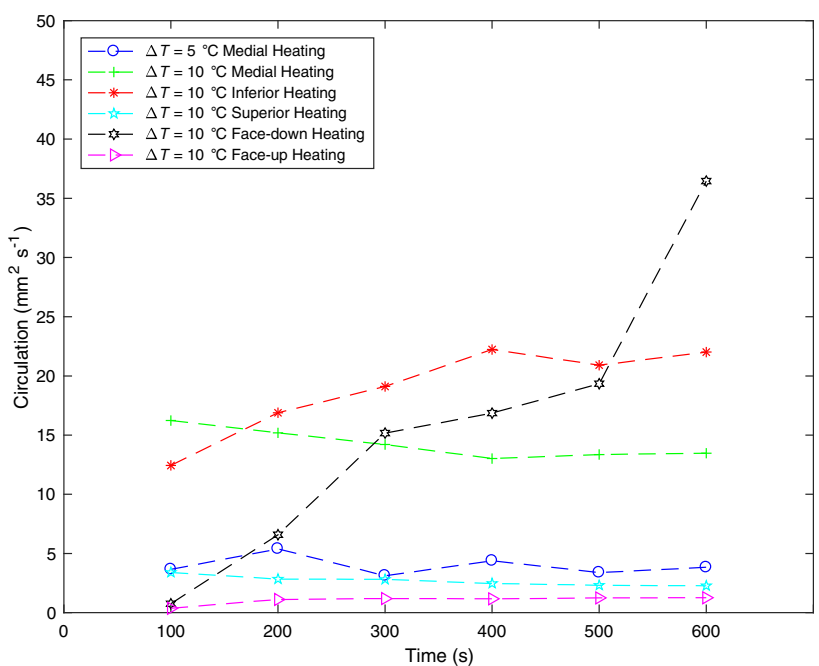

FIGURE 5. Absolute value of circulation calculated from PIV data, which represents the strength of rotational motion, for each heating case in the eye model.

50 min of heating. In contrast, when heating was applied from the inferior position (Fig. 7b), drug gradually started to follow the circulation starting around $20 \mathrm{~min}$ after heating was applied and filled up the entire plane of observation in $50 \mathrm{~min}$. By this time, the drug simulant was able to reach the targeted region. Results from pixel intensity quantification show the same trend. No drug entered the targeted area throughout the entire time when heating from the medial position was applied (Fig. 8a), whereas a substantial amount of drug reached the targeted area when heating was applied from the inferior position. The drug concentration increased significantly by the end of 50 min observation (Fig. 8b).

When the drug simulant was lighter and less viscous than the vitreous humor (Figs. 7c and 7d), it floated in the top region of the eye model after injection. For both medial (Fig. 7c) and inferior positions (Fig. 7d), drug simulant started to move toward the target area along the model wall by the 10 min mark. This circulation motion continued until the end of the observation $(50 \mathrm{~min})$ for both cases, allowing the drug to fill up the entire plane of observation. Similarly, in Fig. 8c and Fig. 8d, as the thermal effect slowly built up, a good amount of drug did reach the targeted region by the end of $50 \mathrm{~min}$. Interestingly, the heating position did not make an observable difference between these two cases.

In summary, when drugs are heavier and more viscous than the vitreous humor, heating from the medial position is not very helpful for facilitating drug mixing in the eye. When drugs are lighter and less viscous than the vitreous humor, both medial and inferior heating 

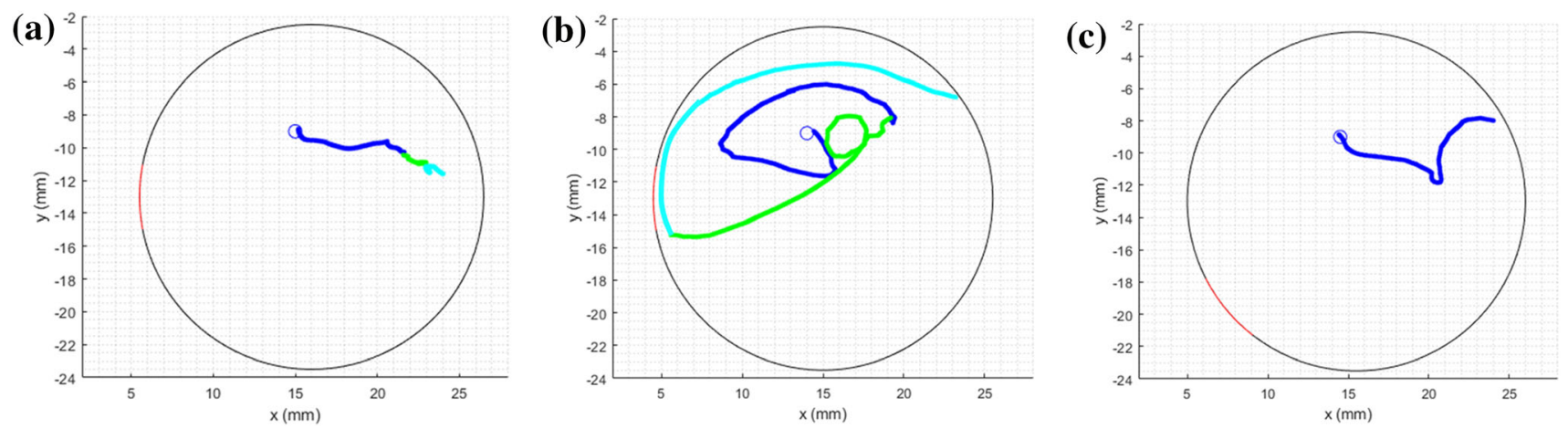

FIGURE 6. Individual particle tracking for three different scenarios. The blue circle indicates the initial position of the particle, the dark blue line indicates particle movements during the first $200 \mathrm{~s}$, the green line indicates particle movements during the second $200 \mathrm{~s}$, and the light blue line indicates particle movements during the last $200 \mathrm{~s}$. Heating position is marked in red along the circular boundary. (a) Medial heating with $\triangle T=5^{\circ} \mathrm{C}$. (b) Medial heating with $\triangle T=10^{\circ} \mathrm{C}$. (c) Inferior heating with $\triangle T=10^{\circ} \mathrm{C}$.

positions are useful and can induce strong drug mixing profiles within an hour. The inferior heating position can induce strong drug mixing regardless of the density of the drug.

\section{DISCUSSION}

In this study, we propose a novel method to thermally induce fluid mixing in the vitreous chamber and present in vitro results for model experiments of the method. We show that the formation of strong circulation by heating can potentially enhance the drug delivery process. Its effectiveness is twofold: it facilitates the drug movement to the back of the eye (which is the location of the macula), and it induces better drug mixing in the vitreous chamber.

Based on observations from the LIF visualization study, if the injected drug is heavier and more viscous than the vitreous humor, drug delivery efficiency can be reduced. However, this could be solved by changing the heating location. Because properties of the vitreous vary a lot among individuals, and also change during the aging process, modifying the temperature difference, heating position, or even some of the physical properties of the anti-VEGF drug accordingly can boost the treatment performance for different individuals. Generally, our results indicate that heating at a greater temperature gradient from an inferior position or from the medial position while face-down could potentially work well for most patients.

A few limitations regarding the experimental design are worth mentioning. First, we visualized a 2D plane of a 3D object, which was sufficient for our purposes because the macula is a small spot located near the center of the back of the retina. The visualized 2D plane went directly through this corresponding location on the eye model. To analyze the drug trajectories in the entire vitreous chamber, a 3D PIV visualization might be useful.

Secondly, coefficients of thermal conductivity of glass $(0.80)$ and air (0.26) are different from that of ocular tissues (cornea and sclera: 0.58 ). The wellknown Penne's bioheat transfer equation is the standard for calculating temperature distribution in living tissues. It has been widely used to numerically analyze heat effects in the human eye. The equation is written as

$$
\nabla \cdot k \nabla T+q_{p}+q_{m}-W c_{b}\left(T-T_{a}\right)=\rho c_{p} \frac{\partial T}{\partial t},
$$

where $k$ is the tissue thermal conductivity, $T$ is the local tissue temperature, $q_{p}$ is the external power deposition rate, $q_{m}$ is metabolism, $T_{a}$ is the arterial temperature, $W$ is the local tissue-blood perfusion rate, $c_{b}$ is the blood specific heat, $c_{p}$ is the tissue specific heat, and $\rho$ is the tissue density.

The model does not describe any convective heat transfer mechanism, but it can offer a basic understanding of what could happen when a heat source is applied to the eye. When the sclera is heated, blood circulation acts as an energy sink $\left(T>T_{\mathrm{a}}\right)$ and carries away the energy. In our study, the overall temperature increase in the eye model was around $2{ }^{\circ} \mathrm{C}$, which is conductive enough for our purpose. However, since air is still less thermally conductive and may not remove energy as efficiently from the system, it could have allowed the mixture in the eye model to heat up more easily. This indicates that for real eye applications, a higher temperature difference is likely to be preferred. A study on the heat tolerance of the sclera by Dewhirst et al. concluded that the threshold temperature for thermal damage is in the range of $59-61{ }^{\circ} \mathrm{C}$ when the sclera sample is heated for $10 \mathrm{~min}^{4}$ A temperature difference of $10{ }^{\circ} \mathrm{C}$ as recommended in our study is 
(a)



(b)

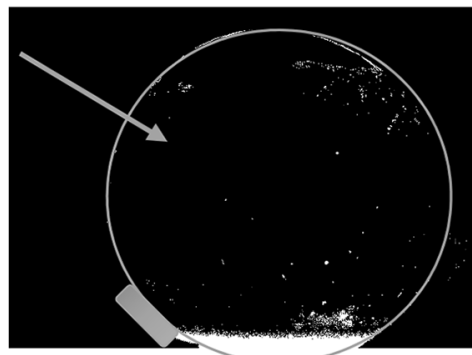

(c)

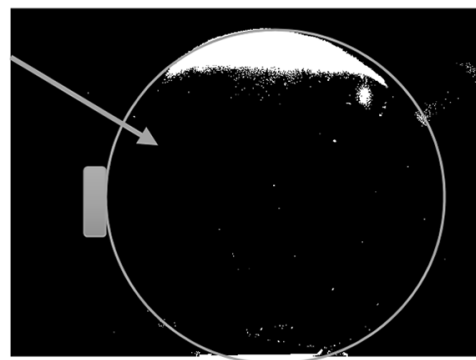

(d)

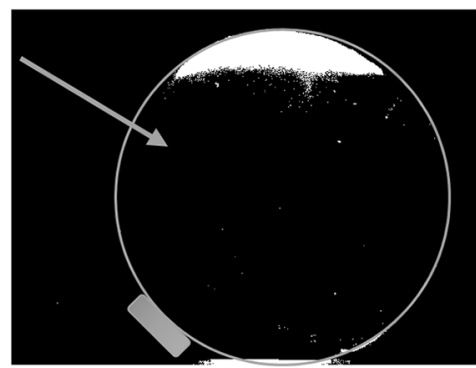

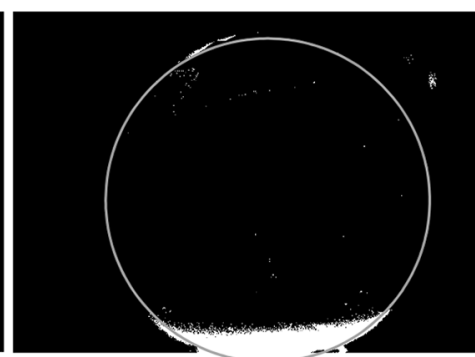
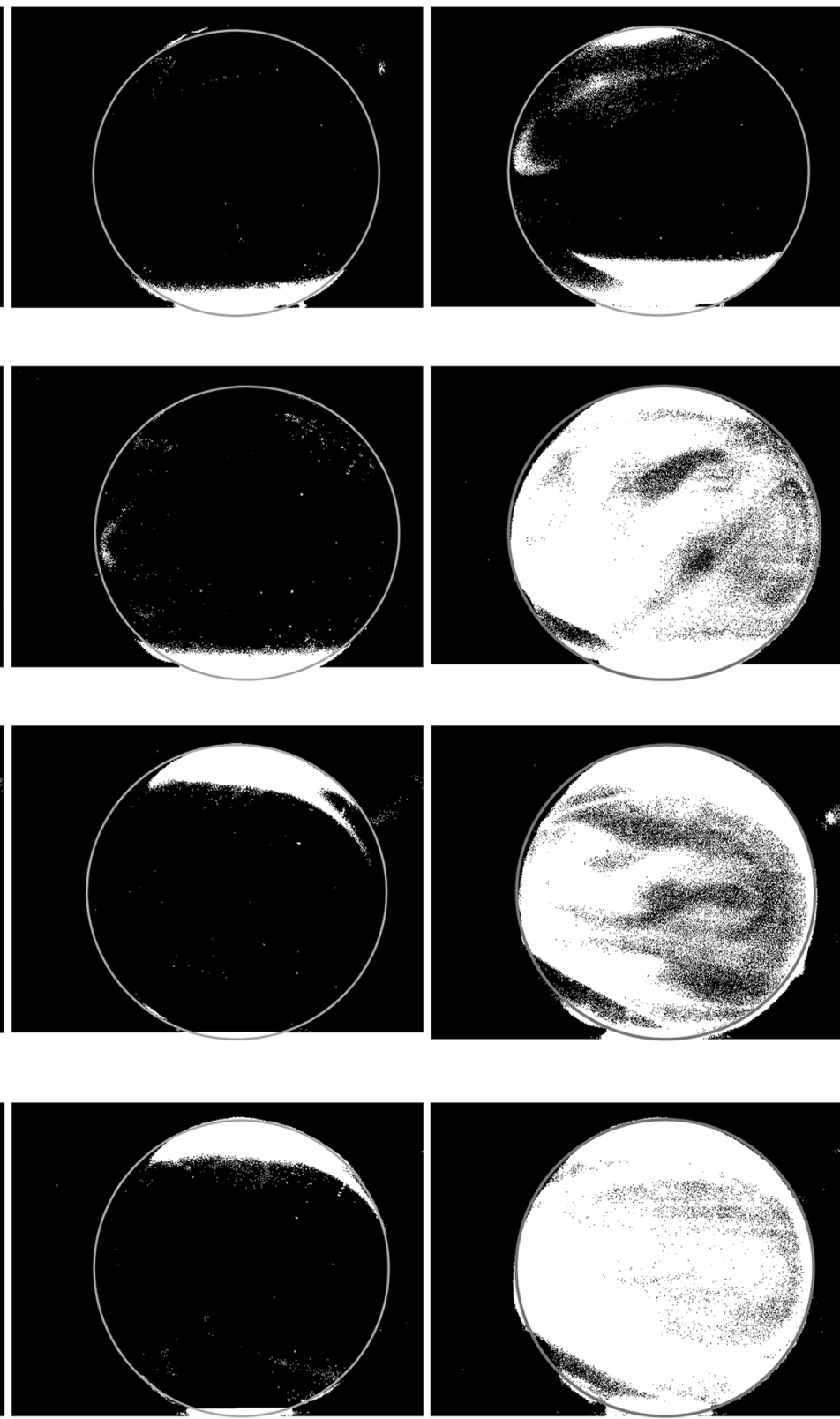

FIGURE 7. Images from laser-induced fluorescence (LIF) visualization at 0,10 , and 50 min after start of heating. The injection was made at 0 min. $\triangle T=10^{\circ} \mathrm{C}$ for all four cases. (a) Adding 33.6 wt $\%$ glycerol drug mixture into 20.2 wt $\%$ glycerol vitreous mixture with medial heating. (b) Adding 33.6 wt\% glycerol drug mixture into 20.2 wt\% glycerol vitreous mixture with inferior heating. (c) Adding 11.2 wt \% glycerol drug mixture into 20.2 wt \% glycerol vitreous mixture with medial heating. (d) Adding 11.2 wt $\%$ glycerol drug mixture into $\mathbf{2 0 . 2}$ wt\% glycerol vitreous mixture with inferior heating. The white circle is artificially labelled in all images to highlight the boundary of the eye model; the arrows and squares in left images indicate the injection spot and heating location, respectively.

well below this limit, which offers more flexibility for applications on real eyes.

Additionally, the refractive index is 1.36 for the working fluid (20.2 wt\% glycerol) and 1.46 for the quartz glass used in this study. Immersing the glass globe in a viewing box that is filled with the working fluid can fix the refractive index issue. However, this is difficult to achieve because it is challenging to ensure the controlled local heating when a glass globe is immersed in the fluid. To understand the potential influence, we repeated the experiment with the face-up heating position with and without a fluid-filled viewing box. We learned that the percentage difference is in the range of $12-20 \%$ and the general vorticity increasing 

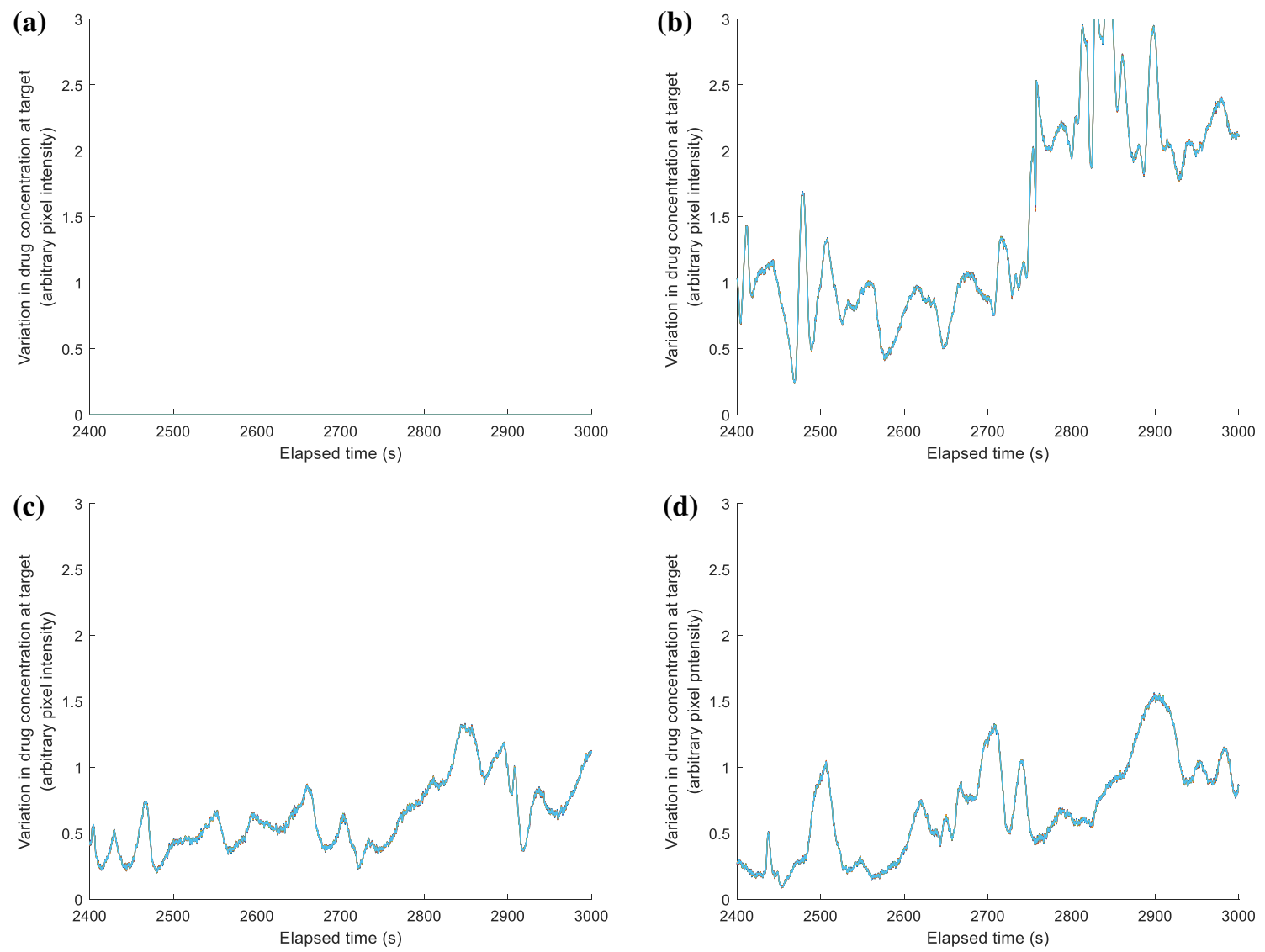

FIGURE 8. Pixel intensity quantification results from laser-induced fluorescence (LIF) visualization for simulations of drug injection. (a) Heavier drug, $\triangle T=10^{\circ} \mathrm{C}$ medial heating at 40-50 min. (b) Heavier drug, $\triangle T=10^{\circ} \mathrm{C}$ inferior heating at $40-50$ min. (c) Lighter drug, $\triangle T=10^{\circ} \mathrm{C}$ medial heating at 40-50 min. (d) Lighter drug, $\triangle T=10^{\circ} \mathrm{C}$ inferior heating at $40-50 \mathrm{~min}$.

trend is consistent. This experimental result confirms that the potential error caused by unmatched refractive index will not change our conclusion.

Looking forward, variations on some important parameters remain to be explored to develop more personalized treatments. Interesting parameters relevant to both heating techniques (such as temperature difference, heating position and contact area) and the morphology of the human eye (such as vitreous humor properties, which change the Prandtl number, and different geometries of the $e^{2} \mathrm{e}^{2}$ ) can be investigated. Additionally, the shear viscosity is assumed to be constant in this study, which could vary with the rate of strain in liquefied vitreous. Therefore, the effects of changing shear viscosity could also be included in future experiments.

Ideally, the thermally induced heating technique can serve as a non-invasive add-on step at the end of the intravitreal injection procedure. Applying a medicalgrade heating pad/probe to the desired location of the eye for a short period of time can promote treatment efficacy in a safe way. Additionally, this research outcome also paves the way for improving eye treatment techniques beyond intravitreal injections. Devices such as eye implants and sustained-release intraocular drug delivery devices can also incorporate this idea to improve their respective treatment performance. We hope that the hundreds of millions of patients who are suffering from ineffective treatments as well as side effects can benefit from this technique in the future.

\section{ELECTRONIC SUPPLEMENTARY MATERIAL}

The online version of this article (https://doi.org/10. 1007/s10439-020-02534-9) contains supplementary material, which is available to authorized users.

\section{ACKNOWLEDGMENTS}

We thank the Charyk Family Foundation for the funding support for this work. We are grateful to Alessio Tamborini for his support in the refractive index experiment, and to Dr. Cong Wang and Dr. Chris Roh for productive discussions. 


\section{REFERENCES}

${ }^{1}$ Balazs, E. A. Functional anatomy of the vitreous. In: Duane's Foundations of Clinical Ophthalmology, Vol. 1, edited by W. Tasman, and E. A. Jaeger. Philadelphia: Lipppincott, 1994, pp. 1-16.

${ }^{2}$ Bonfiglio, A. Investigation of the motion of a viscous fluid in the vitreous cavity induced by eye rotations and implications for drug delivery. Phys. Med. Biol. 58(6):1969, 2013.

${ }^{3}$ Del Amo, E. M., A.-K. Rimpelä, E. Heikkinen, O. K. Kari, E. Ramsay, T. Lajunen, M. Schmitt, L. Pelkonen, M. Bhattacharya, D. Richardson, A. Subrizi, T. Turunen, M. Reinisalo, J. Itkonen, E. Toropainen, M. Casteleijn, H. Kidron, M. Antopolsky, K.-S. Vellonen, M. Ruponen, and A. Urtti. Pharmacokinetic aspects of retinal drug delivery. Prog. Retinal Eye Res. 57:134-185, 2017.

${ }^{4}$ Dewhirst, M. W., B. L. Viglianti, M. Lora-Michiels, M. Hanson, and P. J. Hoopes. Basic principles of thermal dosimetry and thermal thresholds for tissue damage from hyperthermia. Int. J. Hyperthermia 19(3):267-294, 2003.

${ }^{5}$ Eisner, G. Clinical anatomy of the vitreous. In: Duane's Foundations of Clinical Ophthalmology, Vol. 1, edited by W. Tasman, and E. A. Jaeger. Philadelphia: Lippincott, 1994, pp. 17-34.

${ }^{6}$ Haddrill, M. What is age-related macular degeneration? Retrieved from https://www.allaboutvision.com/condition s/amd.htm. (accessed 19 December 2019).

${ }^{7}$ Hogan, M. J., J. A. Alvarado, and J. E. Weddel. Histology of the Human Eye. Philadelphia: Saunders, 1971.

${ }^{8}$ Janoria, K. G., S. Gunda, H. S. Boddu, and A. K. Mitra. Novel approaches to retinal drug delivery. Expert Opin. Drug Deliv. 4(4):371-388, 2007.

${ }^{9}$ Locke, J. C., and W. R. Morton. Further studies of the viscosity of aspirated human vitreous fluid: with special reference to its use in retinal detachment surgery. Trans. Am. Ophthalmol. Soc. 63:129, 1965.

${ }^{10}$ Maurice, D. M., and S. Mishima. Ocular pharmacology. In: Handbook of Experimental Pharmacology, edited by M. Sears. Berlin: Springer, 1984, pp. 16-119.

${ }^{11}$ Milo, R., and R. Phillips. Cell Biology by the Numbers. New York: Garland Science, 2015.

${ }^{12}$ Murthy, K. R., R. Goel, Y. Subbannayya, H. K. Jacob, P. R. Murthy, S. S. Manda, A. H. Patil, R. Sharma, N. A.
Sahasrabuddhe, A. Parashar, B. G. Nair, V. Krishna, T. K. Prasad, H. Gowda, and A. Pandey. Proteomic analysis of human vitreous humor. Clin. Proteom. 11(1):29, 2014.

${ }^{13}$ Purves, D., G. J. Augustine, D. Fitzpatrick, L. C. Katz, A.S. LaMantia, J. O. McNamara, and S. M. Williams (eds.). Anatomy of the Eye. Neuroscience (2nd ed.). Sunderland, MA: Sinauer Associates, 2001.

${ }^{14}$ Remington, L. A., and D. Goodwin. Clinical Anatomy of the Visual System E-Book. Amsterdam: Elsevier Health Sciences, 2011.

${ }^{15}$ Scott, K. S., and J. S. Oliver. Vitreous humor as an alternative sample to blood for the supercritical fluid extraction of morphine and 6-monoacetylmorphine. Med. Sci. Law 39(1):77-81, 1999.

${ }^{16}$ Shah, S. S., L. V. Denham, J. R. Elison, P. S. Bhattacharjee, C. Clement, T. Huq, and J. M. Hill. Drug delivery to the posterior segment of the eye for pharmacologic therapy. Expert Rev. Ophthalmol. 5(1):75-93, 2010.

${ }^{17}$ Stocchino, A., R. Repetto, and J. H. Siggers. Mixing processes in the vitreous chamber induced by eye rotations. Phys. Med. Biol. 55(2):453-467, 2010.

${ }^{18}$ Wessapan, T., and P. Rattanadecho. Heat transfer analysis of the human eye during exposure to sauna therapy. $\mathrm{Nu}$ mer. Heat Transf. A: Appl. 68(5):566-582, 2015.

${ }^{19}$ Whitmore, S. S., E. H. Sohn, K. R. Chirco, A. V. Drack, E. M. Stone, B. A. Tucker, and R. F. Mullins. Complement activation and choriocapillaris loss in early AMD: implications for pathophysiology and therapy. Prog. Retin. Eye Res. 45:1-29, 2015.

${ }^{20}$ Wilson, M. E., and A. W. Scott. How to give intravitreal injections. EyeNet Mag., 2013.

${ }^{21}$ Wong, W. L., X. Su, X. Li, C. M. G. Cheung, R. Klein, C. Y. Cheng, and T. Y. Wong. Global prevalence of age-related macular degeneration and disease burden projection for 2020 and 2040: a systematic review and meta-analysis. Lancet Glob. Health 2(2):e106-e116, 2014.

${ }^{22}$ Yorston, D. Anti-VEGF drugs in the prevention of blindness. Community Eye Health 27(87):44, 2014.

Publisher's Note Springer Nature remains neutral with regard to jurisdictional claims in published maps and institutional affiliations. 